To appear in Physics Letters A

\title{
On the construction of evolution equations admitting a master symmetry
}

\author{
F. FinKEL* \\ Departamento de Física Teórica II \\ Universidad Complutense \\ 28040 Madrid \\ Spain
}

\author{
A.S. FOKAS \\ Department of Mathematics \\ Imperial College \\ London SW7 2BZ \\ United Kingdom
}

May 18, 2001

\begin{abstract}
A method for constructing evolution equations admitting a master symmetry is proposed. Several examples illustrating the method are presented. It is also noted that for certain evolution equations master symmetries can be useful for obtaining new conservation laws from a given one.
\end{abstract}

Mathematics Subject Classification (2000): 35Q53, 35Q58, 37K10

Keywords: master symmetries, integrable evolution equations, conservation laws

*Corresponding author. E-mail: federico@ciruelo.fis.ucm.es 


\section{Introduction}

The concept of master symmetries was introduced in [1]. This concept, which was further developed in [2 [6], is deeply related to the so called bispectral problem [7], as well as to Virasoro algebras [8]. Among the direct applications of master symmetries are the recursive construction of hierarchies of infinitely many symmetries and of infinitely many gradients of conserved densities for nonlinear integrable evolution equations.

In this paper we present an algorithm for constructing evolution equations which are invariant under a given group of scaling transformations and which possess a master symmetry. Moreover, as a further indication of the importance of master symmetries, we note that they can be useful for constructing new conservation laws starting from a given one. We note that the method introduced here can be applied to other types of equations such as the very interesting class of non-linear equations recently introduced in [9].

We first introduce the basic rings used throughout this paper. Then we define symmetries, master symmetries, and scalings.

Definition 1.1 (The basic rings). Let $q(t, \boldsymbol{x}), t \in \mathbb{R}, \boldsymbol{x}=\left(x_{1}, \ldots, x_{n}\right) \in \mathbb{R}^{n}$, be a smooth function of the indicated variables. Let $\partial_{x_{j}}^{-1}$ denote the integral operators $\partial_{x_{j}}^{-1}=\frac{1}{2}\left(\int_{-\infty}^{x_{j}}-\int_{x_{j}}^{\infty}\right), j=1, \ldots, n$. We shall denote by $\mathcal{R}\{q\}$ the ring of smooth functions of $q$ and its $x_{j}$-partial derivatives; $\mathcal{R}\left\{q, \partial^{-1} q\right\}$ will denote the ring of smooth functions of $q$, its $x_{j}$-partial derivatives, and its integrals $\partial_{x_{j}}^{-1}$. If these smooth functions are polynomials, the corresponding rings will be denoted by $\mathcal{P}\{q\}$ and $\mathcal{P}\left\{q, \partial^{-1} q\right\}$. Similarly, if these smooth functions depend explicitly on $\boldsymbol{x}$ and on $t$ we will use the notation $\mathcal{R}\left\{q, \partial^{-1} q, t, \boldsymbol{x}\right\}$.

Definition 1.2 (Symmetries). Let $\Delta(q)$ and $\eta(q)$ belong to $\mathcal{R}\left\{q, \partial^{-1} q, t, \boldsymbol{x}\right\}$. We call $\eta$ a symmetry of the PDE

$$
\Delta(q)=0
$$

iff

$$
\Delta^{\prime} \eta=0 \quad \text { when } \quad \Delta=0
$$

where $\Delta^{\prime}$ denotes the Fréchet derivative of $\Delta$ with respect to q, i.e.,

$$
\Delta^{\prime}(q) \eta(q)=\left.\frac{\partial}{\partial \varepsilon} \Delta(q+\varepsilon \eta)\right|_{\varepsilon=0}
$$




\section{Constructing master symmetries}

Let $q$ satisfy the evolution PDE

$$
q_{t}=K_{1}(q), \quad K_{1}(q) \in \mathcal{R}\left\{q, \partial^{-1} q\right\} .
$$

The definition of a symmetry implies that the function $K_{2}(q) \in \mathcal{R}\left\{q, \partial^{-1} q\right\}$ is a symmetry of this evolution PDE iff

$$
\left[K_{1}(q), K_{2}(q)\right]_{L}=0,
$$

where the Lie commutator $[,]_{L}$ is defined by

$$
[A(q), B(q)]_{L}=A^{\prime} B-B^{\prime} A .
$$

We note that since $K_{1}$ and $K_{2}$ are $x_{j}$-independent they commute with $q_{x_{j}}$, i.e.

$$
\left[q_{x_{j}}, K_{1}\right]_{L}=\left[q_{x_{j}}, K_{2}\right]_{L}=0 .
$$

Thus for evolution equations the question of constructing symmetries which are independent of $x_{j}$ and of $t$, reduces to the question of constructing an Abelian Lie algebra with elements $\left\{q_{x_{j}}, K_{1}, K_{2}\right\}$. Such a construction can be carried out using a master symmetry.

Definition 1.3 (Master symmetries). Suppose that $K_{1}(q), K_{2}(q) \in \mathcal{R}\left\{q, \partial^{-1} q\right\}$ commute, i.e., suppose that they satisfy Eq. (11.5). We call $\tau(q) \in \mathcal{R}\left\{q, \partial^{-1} q, \boldsymbol{x}\right\}$ a master symmetry associated with $q_{x_{j}}, K_{1}$, and $K_{2}$, iff

$$
\left[q_{x_{j}}, \tau\right]_{L}=c_{1} K_{1}, \quad\left[K_{1}, \tau\right]_{L}=c_{2} K_{2},
$$

where $c_{1}$ and $c_{2}$ are constants.

This definition implies that the Abelian algebra generated by $\left\{q_{x_{j}}, K_{1}, K_{2}\right\}$ is constructed solely from a master symmetry.

The existence of a master symmetry implies the existence of a generalized symmetry $K_{2}$. 1 Although in all known examples the recursive use of $\tau$,

$$
\left[K_{j}, \tau\right]_{L}=c_{j} K_{j+1},
$$

generates infinitely many symmetries, their existence does not follow from Definition 1.3. However, it was conjectured in [10] that the existence of one generalized symmetry implies infinitely many symmetries. This conjecture was shown to be true

\footnotetext{
${ }^{\dagger}$ It is called generalized in juxtaposition to Lie point because either it depends nonlinearly on first-order derivatives or it involves higher-order derivatives, see [5] for further discussion.
} 
in [11] for a large class of evolution equations in one space dimension. This provides further support for the usefulness of constructing master symmetries, since they guarantee the existence of at least one generalized symmetry. In what follows we present such a construction based on the notion of scaling:

Definition 1.4 (Scalings). Suppose that $K(q) \in \mathcal{R}\left\{q, \partial^{-1} q, \boldsymbol{x}\right\}$ is invariant up to a multiplicative constant under the transformation $q \mapsto \alpha^{\lambda} q, x_{j} \mapsto \alpha^{\lambda_{j}} x_{j}$, i.e.

$$
K\left(\alpha^{\lambda} q\left(t, \alpha^{\lambda_{1}} x_{1}, \ldots, \alpha^{\lambda_{n}} x_{n}\right)\right)=\mu K\left(q\left(t, x_{1}, \ldots, x_{n}\right)\right),
$$

where $\alpha, \lambda, \lambda_{j}, \mu$ are constants. Then we say that $K(q)$ admits the scaling $\tau_{0}(q)$, where $\tau_{0}$ is defined by

$$
\tau_{0}(q)=\lambda q-\sum_{1}^{n} \lambda_{j} x_{j} q_{x_{j}}
$$

If the constant $\mu$ equals one, we say that $K(q)$ is an absolute invariant of the scaling $\tau_{0}$.

It is shown in [⿴囗 hat if $K(q) \in \mathcal{R}\left\{q, \partial^{-1} q, \boldsymbol{x}\right\}$ admits the scaling $\tau_{0}(q)$, then

$$
\left[K, \tau_{0}\right]_{L}=c K
$$

where $c$ is a constant.

Observation 1.1 (Candidates for master symmetries). Suppose that $K_{1}(q) \in \mathcal{R}\left\{q, \partial^{-1} q\right\}$ admits the scaling $\tau_{0}(q)$ defined by Eq. (1.9). Assume that there exists a function $f(q) \in \mathcal{R}\left\{q, \partial^{-1} q, \boldsymbol{x}\right\}$ such that $\tau_{0}$ can be written in the form

$$
\tau_{0}=\left[q_{x_{j}}, f\right]_{L}
$$

Define $\tau(q) \in \mathcal{R}\left\{q, \partial^{-1} q, \boldsymbol{x}\right\}$ by

$$
\tau=\left[K_{1}, f\right]_{L}+\tau_{1}
$$

where $\tau_{1}(q) \in \mathcal{R}\left\{q, \partial^{-1} q, x_{1}, \ldots, x_{j-1}, x_{j+1}, \ldots, x_{n}\right\}$ is an arbitrary $x_{j}$-independent function. Then $\tau$ satisfies

$$
\left[q_{x_{j}}, \tau\right]_{L}=c_{1} K_{1}
$$

Indeed, since $\tau_{1}$ is $x_{j}$-independent, it commutes with $q_{x_{j}}$; thus

$$
\left[q_{x_{j}}, \tau\right]_{L}=\left[q_{x_{j}},\left[K_{1}, f\right]_{L}\right]_{L}=\left[K_{1},\left[q_{x_{j}}, f\right]_{L}\right]_{L}-\left[f,\left[q_{x_{j}}, K_{1}\right]_{L}\right]_{L}
$$


where we have used the fact that $[,]_{L}$ satisfies the Jacobi identity. Since $K_{1}$ is $x_{k^{-}}$ independent for all $k$ and admits the scaling $\tau_{0}$, Eq. (1.14) becomes

$$
\left[q_{x_{j}}, \tau\right]_{L}=\left[K_{1}, \tau_{0}\right]_{L}=c_{1} K_{1}
$$

The above observation suggests the following algorithm for constructing equations which admit a given scaling $\tau_{0}$ and a master symmetry:

Construction 1.1 (Construction of master symmetries).

1. Start with a general function $K_{1}(q) \in \mathcal{R}\left\{q, \partial^{-1} q\right\}$ which remains invariant up to a multiplicative constant under the transformation $q \mapsto \alpha^{\lambda} q, x_{j} \mapsto \alpha^{\lambda_{j}} x_{j}$ (see Eq. (1.8)). Let the associated scaling $\tau_{0}$ be defined by Eq. (1.9). The function $K_{1}(q)$ involves some absolute invariants $b_{j}(q) \in \mathcal{R}\left\{q, \partial^{-1} q\right\}$ of the scaling $\tau_{0}$.

2. Find a function $f(q) \in \mathcal{R}\left\{q, \partial^{-1} q, \boldsymbol{x}\right\}$ such that Eq. (1.11) is satisfied.

3. Define $\tau(q) \in \mathcal{R}\left\{q, \partial^{-1} q, \boldsymbol{x}\right\}$ by $E q$. (1.12) and $K_{2}(q)$ by

$$
K_{2}=\left[K_{1}, \tau\right]_{L}
$$

If there exist absolute invariants $b_{j}(q)$ and a function $\tau_{1}(q)$ such that $K_{2} \in \mathcal{R}\left\{q, \partial^{-1} q\right\}$ and $\left[K_{1}, K_{2}\right]_{L}=0$, then $\tau$ is a master symmetry associated with $q_{x_{j}}, K_{1}, K_{2}$.

We note that the requirement $K_{2} \in \mathcal{R}\left\{q, \partial^{-1} q\right\}$ is automatically satisfied in one space dimension $(n=1)$. Indeed,

$$
\left[q_{x}, K_{2}\right]_{L}=\left[q_{x},\left[K_{1}, \tau\right]_{L}\right]_{L}=\left[K_{1},\left[q_{x}, \tau\right]_{L}\right]_{L}-\left[\tau,\left[q_{x}, K_{1}\right]_{L}\right]_{L}=0
$$

where we have used Eq. (1.13) and the fact that $K_{1}$ commutes with $q_{x}$.

Example 1.1 The most general third-order function $K_{1}(q) \in \mathcal{P}\{q\}$ which remains invariant up to a multiplicative constant under the scaling

$$
q \mapsto \alpha^{-1} q, \quad x \mapsto \alpha^{2} x
$$

is given by

$$
K_{1}=q_{3}+3 q^{2} q_{2}+\beta_{1} q q_{1}^{2}+\beta_{2} q^{4} q_{1}+\beta_{3} q^{7}
$$

where throughout the paper

$$
q_{j}=\partial_{x}^{j} q
$$

and $\beta_{1}, \beta_{2}, \beta_{3}$ are constants. The scaling (1.16) implies that $\tau_{0}=q+2 x q_{x}$. The class of solutions of the equation $\tau_{0}=\left[q_{x}, f\right]_{L}$ depend on the form of $f$. If $f \in \mathcal{R}\left\{q, \partial^{-1} q, x\right\}$, then

$$
f=x q+x^{2} q_{1}+g(q)
$$

\footnotetext{
${ }^{\ddagger}$ Note that any absolute invariant of the scaling (1.16) in $\mathcal{P}\{q\}$ is necessarily a constant.
} 
where $g$ is an arbitrary function in $\mathcal{R}\left\{q, \partial^{-1} q\right\}$. We take $g=0$, define $\tau$ by Eq. (1.12) with $\tau_{1}=0$, and determine $K_{2}$ from Eq. (1.15). A straightforward computation shows that $K_{2}$ commutes with $K_{1}$ if and only if

$$
\beta_{1}=9, \quad \beta_{2}=3, \quad \beta_{3}=0 .
$$

We recover in this way the Ibragimov-Shabat equation [12, 13]

$$
q_{t}=q_{3}+3 q^{2} q_{2}+9 q q_{1}^{2}+3 q^{4} q_{1}
$$

this equation admits the master symmetry

$$
\tau=2 x\left(q_{3}+3 q^{2} q_{2}+9 q q_{1}^{2}+3 q^{4} q_{1}\right)+3 q_{2}+10 q^{2} q_{1}+q^{5} .
$$

We note that the master symmetry of Burgers equation, both master symmetries of the Kadomtsev-Petviashvili (KP) equation, and the master symmetries of several other equations (see Eqs. (2.7), (2.14), (2.17), (2.11), (2.20)) can be obtained through Construction 1.1.

\section{Master symmetries and conservation laws}

It is well known 14 that certain symmetries can be used to construct a new conservation law from a given one. However, not all symmetries can be used for this purpose since some symmetries yield trivial conservation laws. For example, the Korteweg-de Vries (KdV) equation

$$
\partial_{t} q=\partial_{x}\left(q_{2}+3 q^{2}\right)
$$

is in a form of a conservation law and also it admits infinitely many symmetries $K_{j} \in$ $\mathcal{P}\{q\}$. If one starts with the conservation law (1.20), all these symmetries yield a trivial conservation law.

It will be shown in Section 3 that starting from (1.20), the time-dependent generalized symmetry associated with the master symmetry of the KdV equation can be used to generate a hierarchy of nontrivial conservation laws.

\section{Constructing master symmetries}

Example 2.1 The most general second-order function $K_{1} \in \mathcal{P}\{q\}$ which remains invariant up to a multiplicative constant under the scaling

$$
q \mapsto \alpha^{-1} q, \quad x \mapsto \alpha x
$$


is given by

$$
K_{1}=q_{2}+2 q q_{1}+\beta q^{3} .
$$

The above scaling implies that

$$
\tau_{0}=q+x q_{1}
$$

The general solution of Eq. (1.11) in $\mathcal{R}\left\{q, \partial^{-1} q, x\right\}$ is given by

$$
f=x q+\frac{x^{2}}{2} q_{1}+g(q),
$$

where $g$ is an arbitrary function in $\mathcal{R}\left\{q, \partial^{-1} q\right\}$. Define $\tau$ by Eq. (1.12) with $g=\tau_{1}=0$, i.e.,

$$
\tau=\left[K_{1}, f\right]_{L}=2 x K_{1}+3 q_{1}+2 q^{2} .
$$

A simple computation shows that the function $K_{2}$ defined by

$$
K_{2}=\left[K_{1}, \tau\right]_{L}=4\left(q_{3}+3 q q_{2}+3 q_{1}^{2}+(3+\beta) q^{2} q_{1}+\frac{3 \beta}{2} q^{4}\right)
$$

commutes with $K_{1}$ iff $\beta=0$. In summary, the Burgers equation

$$
q_{t}=q_{2}+2 q q_{1}
$$

admits the master symmetry

$$
\tau=2 x\left(q_{2}+2 q q_{1}\right)+3 q_{1}+2 q^{2} .
$$

Example 2.2 Consider the function $K_{1} \in \mathcal{R}\{q\}$ given by

$$
K_{1}=q_{3}+3 b_{1}(q) \frac{q_{2}^{2}}{q_{1}}+3 b_{2}(q) q_{1} q_{2}+b_{3}(q) q_{1}^{3},
$$

where $b_{j}(q)$ are arbitrary smooth functions of $q$. We assume that $b_{1}(q)$ is not identically zero. The function $K_{1}$ remains invariant up to a multiplicative constant under the scaling $q \mapsto q, x \mapsto \alpha x$; this corresponds to the function $\tau_{0}=x q_{1}$. The general solution of Eq. (1.11) in $\mathcal{R}\left\{q, \partial^{-1} q, x\right\}$ is of the form

$$
f=\frac{x^{2}}{2} q_{1}+g(q)
$$

where $g \in \mathcal{R}\left\{q, \partial^{-1} q\right\}$. Define $\tau$ by Eq. (1.12) with $g=\tau_{1}=0$, i.e.,

$$
\tau=\left[K_{1}, f\right]_{L}=3\left(x K_{1}+\left(1+2 b_{1}\right) q_{2}+b_{2} q_{1}^{2}\right) .
$$


A straightforward computation shows that the function $K_{2}$ defined by Eq. (1.15) commutes with $K_{1}$ iff

$$
b_{1}=-\frac{1}{4}, \quad b_{2}=b(q), \quad b_{3}=b(q)^{2}+2 b^{\prime}(q),
$$

where $b(q)$ is an arbitrary smooth function of $q$, and $b^{\prime}(q)=\mathrm{d} b / \mathrm{d} q$. In summary, the evolution equation

$$
q_{t}=q_{3}-\frac{3 q_{2}^{2}}{4 q_{1}}+3 b q_{1} q_{2}+\left(b^{2}+2 b^{\prime}\right) q_{1}^{3}
$$

admits the master symmetry

$$
\tau=x\left(q_{3}-\frac{3 q_{2}^{2}}{4 q_{1}}+3 b q_{1} q_{2}+\left(b^{2}+2 b^{\prime}\right) q_{1}^{3}\right)+\frac{1}{2} q_{2}+b q_{1}^{2} .
$$

We note that (2.10) can be obtained from the equation [15]

$$
u_{t}=u_{3}-\frac{3 u_{2}^{2}}{4 u_{1}}
$$

by the transformation

$$
u=\beta_{1} \int^{q} \mathrm{e}^{2 \int^{s} b(r) \mathrm{d} r} \mathrm{~d} s+\beta_{2}
$$

where $\beta_{1}$ and $\beta_{2}$ are constants.

Example 2.3 Consider the KP equation in the variable $q(t, x, y)$,

$$
q_{t}=K_{1}, \quad K_{1}=q_{x x x}+6 q q_{x}+3 \partial_{x}^{-1} q_{y y} .
$$

It is well-known that the KP equation admits infinitely many symmetries and conservation laws. The function $K_{1}$ defining the KP equation admits the scaling

$$
\tau_{0}=2 q+x q_{x}+2 y q_{y} .
$$

It may be easily verified that the function $f \in \mathcal{R}\{q, \boldsymbol{x}\}$ given by

$$
f=y\left(2 q+x q_{x}+y q_{y}\right)
$$

satisfies Eq. (1.11) for $q_{y}$, i.e.,

$$
\tau_{0}=\left[q_{y}, f\right]_{L} .
$$

The function $\tau \in \mathcal{R}\left\{q, \partial^{-1} q, \boldsymbol{x}\right\}$ defined by Eq. (1.12) with $\tau_{1}=0$,

$$
\tau=\left[K_{1}, f\right]_{L}=3\left(y K_{1}+2 x q_{y}+4 \partial_{x}^{-1} q_{y}\right),
$$


is a master symmetry of the KP equation [3, 16]. Indeed, the function $K_{2} \in \mathcal{P}\left\{q, \partial^{-1} q\right\}$ defined by

$$
K_{2}=\left[K_{1}, \tau\right]_{L}=36\left(q_{x x y}+4 q q_{y}+2 q_{x} \partial_{x}^{-1} q_{y}+\partial_{x}^{-2} q_{y y y}\right)
$$

commutes with $K_{1}$.

The alternative equation

$$
\tau_{0}=\left[q_{x}, \tilde{f}\right]_{L}
$$

also leads to a master symmetry of the KP equation. Consider the solution of this equation given by

$$
\tilde{f}=x\left(2 q+\frac{1}{2} x q_{x}+2 y q_{y}\right)
$$

Define $\tilde{\tau}_{1} \in \mathcal{R}\left\{q, \partial^{-1} q, y\right\}$ by

$$
\tilde{\tau}_{1}=6 y\left(q_{x x y}+6 q q_{y}+4 q_{x} \partial_{x}^{-1} q_{y}+3 \partial_{x}^{-2} q_{y y y}\right)+3\left(q_{x x}+4 q^{2}+2 q_{x} \partial_{x}^{-1} q+11 \partial_{x}^{-2} q_{y y}\right) .
$$

Then the function $\tilde{\tau} \in \mathcal{R}\left\{q, \partial^{-1} q, \boldsymbol{x}\right\}$ defined by Eq. (1.12), i.e.,

$$
\tilde{\tau}=\left[K_{1}, \tilde{f}\right]_{L}+\tilde{\tau}_{1}=3 x K_{1}+\frac{1}{3} y K_{2}+6\left(2 q_{x x}+4 q^{2}+q_{x} \partial_{x}^{-1} q+3 \partial_{x}^{-2} q_{y y}\right)
$$

is a master symmetry of the KP equation 3 . 3 Indeed, it may verified that the function

$$
\begin{aligned}
& \tilde{K}_{2}=\left[K_{1}, \tilde{\tau}\right]_{L}=9\left(q_{x x x x x}+10 q q_{x x x}+20 q_{x} q_{x x}+10 q_{x y y}+30 q^{2} q_{x}+10 q_{x} \partial_{x}^{-2} q_{y y}\right. \\
&\left.+20 q_{y} \partial_{x}^{-1} q_{y}+20 q \partial_{x}^{-1} q_{y y}+10 \partial_{x}^{-1}\left(q q_{y y}\right)+10 \partial_{x}^{-1} q_{y}^{2}+5 \partial_{x}^{-3} q_{y y y y}\right)
\end{aligned}
$$

commutes with $K_{1}$. We note that

$$
K_{3}=\left[K_{2}, \tau\right]_{L}=12 \tilde{K}_{2}
$$

In general, the master symmetry $\tilde{\tau}$ generates the odd time-independent generalized symmetries $K_{2 j+1}$ of the KP hierarchy $K_{j+1}=\left[K_{j}, \tau\right]_{L}$.

Example 2.4 The function $K_{1} \in \mathcal{P}\{q\}$ given by

$$
K_{1}=q_{7}+7 q q_{5}+14 q_{1} q_{4}+21 q_{2} q_{3}+14 q^{2} q_{3}+42 q q_{1} q_{2}+7 q_{1}^{3}+\frac{28}{3} q^{3} q_{1}
$$

is the first time-independent higher-order symmetry of the Sawada-Kotera (SK) equation [17]

$$
q_{t}=\tilde{K}_{1}, \quad \tilde{K}_{1}=q_{5}+5 q q_{3}+5 q_{1} q_{2}+5 q^{2} q_{1}
$$

\footnotetext{
$\S$ Notice that $\tilde{\tau}$ reduces to the master symmetry of the $\mathrm{KdV}$ equation if $q$ does not depend on $y$.
} 
The function $K_{1}$ in Eq. (2.18) admits the scaling

$$
\tau_{0}=q+\frac{1}{2} x q_{1} .
$$

A straightforward computation shows that the function $f \in \mathcal{R}\{q, x\}$ given by

$$
f=x q+\frac{1}{4} x^{2} q_{1}
$$

solves Eq. (1.11). Define $\tau_{1} \in \mathcal{R}\left\{q, \partial^{-1} q\right\}$ by

$$
\frac{2}{7} \tau_{1}=3 q_{6}+\tilde{K}_{1} \partial_{x}^{-1} q+22 q q_{4}+32 q_{1} q_{3}+17 q_{2}^{2}+38 q^{2} q_{2}+43 q q_{1}^{2}-5 q_{1} \partial_{x}^{-1} q_{1}^{2}+\frac{5}{3} q_{1} \partial_{x}^{-1} q^{3}+\frac{16}{3} q^{4} .
$$

Then the function $\tau \in \mathcal{R}\left\{q, \partial^{-1} q, x\right\}$ determined by Eq. (1.12), i.e.,

$$
\begin{aligned}
\tau=\left[K_{1}, f\right]_{L}+\tau_{1}=\frac{7}{2}( & x K_{1}+\tilde{K}_{1} \partial_{x}^{-1} q+8 q_{6}+46 q q_{4}+75 q_{1} q_{3}+44 q_{2}^{2}+68 q^{2} q_{2} \\
+ & \left.79 q q_{1}^{2}-5 q_{1} \partial_{x}^{-1} q_{1}^{2}+\frac{5}{3} q_{1} \partial_{x}^{-1} q^{3}+8 q^{4}\right),
\end{aligned}
$$

is a master symmetry associated with $q_{1}, K_{1}$, and $K_{2}=\left[K_{1}, \tau\right]_{L}$. In fact, the master symmetry $\tau$ generates two hierarchies of infinitely many symmetries of the SK equation [18], namely

$$
K_{j+1}=\left[K_{j}, \tau\right]_{L}, \quad \tilde{K}_{j+1}=\left[\tilde{K}_{j}, \tau\right]_{L}, \quad j \geq 1 .
$$

\section{Master symmetries and conservation laws}

In this section we shall show that for certain equations the master symmetries can be useful for constructing new conservation laws from a given one. We first formally define conservation laws and trivial conservation laws. Then we recall a well-known result [14] on the action of a symmetry on a conservation law.

Definition 3.1 (Conservation laws). We say that Eq. (1.1) admits the conservation law characterized by $\left\{T_{j}(q)\right\}_{0}^{n}$, where $T_{j} \in \mathcal{R}\left\{q, \partial^{-1} q, \boldsymbol{x}\right\}, j=0, \ldots, n$, iff

$$
D_{t} T_{0}+\sum_{j=1}^{n} D_{j} T_{j}=0 \quad \text { when } \quad \Delta=0,
$$

where $D_{t}$ and $D_{j}$ denote total differentiation with respect to $t$ and $x_{j}$, i.e.,

$$
D_{t}=\frac{\partial}{\partial t}+q_{t} \frac{\partial}{\partial q}+\cdots, \quad D_{j}=\frac{\partial}{\partial x_{j}}+q_{x_{j}} \frac{\partial}{\partial q}+\cdots .
$$


The component $T_{0}(q)$ of a conservation law is called the conserved density. The vector formed by the remaining components $\left(T_{1}(q), \ldots, T_{n}(q)\right)$ is called the flux. A conservation law is local if $T_{j}(q) \in \mathcal{R}\{q, \boldsymbol{x}\}, j=0, \ldots, n$.

Definition 3.2 (Trivial conservation laws). A conservation law is trivial if there exist smooth functions $Q_{j k} \in \mathcal{R}\left\{q, \partial^{-1} q, \boldsymbol{x}\right\}, j, k=0, \ldots, n$, such that $Q_{j k}=-Q_{k j}$ and

$$
T_{j}=D_{t} Q_{j 0}+\sum_{k=1}^{n} D_{k} Q_{j k}, \quad j=0, \ldots, n, \quad \text { when } \quad \Delta=0 .
$$

A trivial conservation law will be denoted as $\left\{T_{j}\right\}_{0}^{n} \sim 0$. Two conservation laws are equivalent if they differ by a trivial conservation law.

The Definitions 1.2 and 3.1 of symmetries and conservation laws imply:

Observation 3.1 (Symmetries and conservation laws). Assume that the PDE (1.1) possesses a symmetry $\eta(q) \in \mathcal{R}\left\{q, \partial^{-1} q, \boldsymbol{x}\right\}$ and a conservation law characterized by $\left\{T_{j}(q)\right\}_{0}^{n}, T_{j} \in \mathcal{R}\left\{q, \partial^{-1} q, \boldsymbol{x}\right\}$, see Definitions 1.2 and 3.1. Then this equation also possesses a conservation law characterized by $\left\{T_{j}^{\prime}(q) \eta(q)\right\}_{0}^{n}$.

Indeed, it follows from Definitions 1.2 and 3.1 that

$$
\left.\frac{\partial}{\partial \varepsilon} D_{t} T_{0}(q+\varepsilon \eta)\right|_{\varepsilon=0}+\left.\frac{\partial}{\partial \varepsilon} \sum_{j=1}^{n} D_{j} T_{j}(q+\varepsilon \eta)\right|_{\varepsilon=0}=0,
$$

which implies the desired result.

This observation provides an effective way to compute conserved densities for selfadjoint linear systems [14].

Example 3.1 Let $q$ satisfy,

$$
q_{t t}=q_{x x}+q_{y y} .
$$

This equation admits the conservation law characterized by

$$
T_{0}(q)=q_{t} q_{x}, \quad T_{1}(q)=-q q_{y y}-\frac{1}{2}\left(q_{t}^{2}+q_{x}^{2}+q_{y}^{2}\right), \quad T_{2}(q)=q q_{x y} .
$$

Consider the Lorentz-boost symmetry $\eta(q)=x q_{t}+t q_{x}$. Then

$$
\left\{T_{j}^{\prime} \eta\right\}_{0}^{2} \sim\left\{x q_{x} q_{y y}+\frac{1}{2}\left(q_{t}^{2}+q_{x}^{2}\right),-q_{t}\left(q_{x}+x q_{y y}\right), x\left(q_{t} q_{x y}-q_{x} q_{t y}\right)\right\}
$$


is also a conservation law of equation (3.5). We have subtracted here the trivial conservation law determined by (see Eq. (3.3))

$$
Q_{01}=t q_{t} q_{x}+\frac{x}{2}\left(q_{t}^{2}+q_{x}^{2}\right), \quad Q_{02}=0, \quad Q_{12}=-q\left(t q_{x y}+x q_{t y}\right) .
$$

We note that if $T_{j}(q), j=0, \ldots, n$, are invariant with respect to the symmetry $\eta(q)$ under consideration then $\left\{T_{j}^{\prime} \eta\right\}_{0}^{n} \sim 0$, thus the Observation 3.1 does not yield a new conservation law.

Example 3.2 Consider the KdV equation (1.20). The KdV equation admits infinitely many symmetries $K_{j} \in \mathcal{P}\{q\}$ and local conservation laws. The KdV equation is in a form of a conservation law, where

$$
T_{0}(q)=q, \quad T_{1}(q)=-q_{2}-3 q^{2} .
$$

However, since $\left\{T_{0}^{\prime} K_{j}, T_{1}^{\prime} K_{j}\right\} \sim 0$ none of the symmetries $K_{j}$ yield new (nontrivial) conservation laws.

The KdV equation also possesses $x, t$-dependent symmetries $\sigma_{j}(q) \in \mathcal{R}\left\{q, \partial^{-1} q, t, x\right\}$. These symmetries are intimately related to the master symmetry, see the Appendix. In particular, a time-dependent symmetry of the $\mathrm{KdV}$ equation is given by

$$
\sigma=t K_{2}+\tau
$$

where

$$
\tau=\frac{1}{3}\left(x K_{1}+4 q_{2}+8 q^{2}+2 q_{1} \partial_{x}^{-1} q\right)
$$

is the master symmetry of the KdV equation and

$$
K_{2}=\left[K_{1}, \tau\right]_{L}=q_{5}+10 q q_{3}+20 q_{1} q_{2}+30 q^{2} q_{1}
$$

is its first generalized time-independent symmetry. The symmetry $\sigma$ gives rise to the conservation law

$$
T_{0}^{(1)}(q)=q^{2}, \quad T_{1}^{(1)}(q)=-2 q q_{2}+q_{1}^{2}-4 q^{3} .
$$

Indeed, on solutions of the $\mathrm{KdV}$ equation we have

$$
\left\{T_{0}^{\prime} \sigma, T_{1}^{\prime} \sigma\right\}=\left\{T_{0}^{(1)}, T_{1}^{(1)}\right\}+\left\{D_{x} Q,-D_{t} Q\right\}
$$

where

$$
Q=t\left(q_{4}+10 q q_{2}+5 q_{1}^{2}+10 q^{3}\right)+x\left(\frac{1}{3} q_{2}+q^{2}\right)+q_{1}+\frac{2}{3} q \partial_{x}^{-1} q
$$


It should emphasized that the conserved density $T_{0}^{(1)}$ of the conservation law (3.9) can be generated solely by $\tau$, up to a total $x$-derivative. The recursive application of the Fréchet derivative with respect to $\sigma$ can be used to obtain the well-known sequence of conservation laws associated with the KdV equation:

$$
\begin{aligned}
\left\{T_{0}^{(2)}, T_{1}^{(2)}\right\} & =\left\{-q_{1}^{2}+2 q^{3}, 2 q_{1} q_{3}-q_{2}^{2}-6 q^{2} q_{2}+12 q q_{1}^{2}-9 q^{4}\right\} \\
\left\{T_{0}^{(3)}, T_{1}^{(3)}\right\} & =\left\{q_{2}^{2}-10 q q_{1}^{2}+5 q^{4},-2 q_{2} q_{4}+q_{3}^{2}+20 q q_{1} q_{3}-16 q q_{2}^{2}-10 q_{1}^{2} q_{2}-20 q^{3} q_{2}\right. \\
& \left.+90 q^{2} q_{1}^{2}-24 q^{5}\right\} \\
\left\{T_{0}^{(4)}, T_{1}^{(4)}\right\} & =\left\{-q_{3}^{2}+14 q q_{2}^{2}-70 q^{2} q_{1}^{2}+14 q^{5}, 2 q_{3} q_{5}-q_{4}^{2}-28 q q_{2} q_{4}+20 q q_{3}^{2}+28 q_{1} q_{2} q_{3}\right. \\
& \left.+140 q^{2} q_{1} q_{3}-2 q_{2}^{3}-154 q^{2} q_{2}^{2}-140 q q_{1}^{2} q_{2}-70 q^{4} q_{2}+35 q_{1}^{4}+560 q^{3} q_{1}^{2}-70 q^{6}\right\}
\end{aligned}
$$

where we have omitted an irrelevant global constant and a trivial conservation law. The time-dependent symmetry $\sigma$ thus plays for conservation laws the same role as the adjoint of the recursion operator for their conserved gradients; see Ref. [5]. The formalism based on the time-dependent symmetry $\sigma$ associated with the master symmetry is more convenient, because it provides explicit expressions for the conserved densities $T_{0}^{(k)}$, and what is more important, for the corresponding fluxes $T_{1}^{(k)}$.

Remark 3.1 In some cases, the time-dependent symmetry associated with the master symmetry yields trivial conservation laws. For example, the Burgers equation (2.6) is also in the form of a conservation law, with

$$
T_{0}(q)=q, \quad T_{1}(q)=-q_{1}-q^{2}
$$

Unlike the $\mathrm{KdV}$ equation, the Burgers equation is diffusive thus does not possess infinitely many conservation laws. In this case $\sigma=t K_{2}+\tau$, where $\tau$ and $K_{2}$ are respectively given by Eqs. (2.7) and (2.5) with $\beta=0$, yields a trivial conservation law, namely

$$
\left\{T_{0}^{\prime} \sigma, T_{1}^{\prime} \sigma\right\}=\left\{D_{x} Q,-D_{t} Q\right\}
$$

where

$$
Q=4 t\left(q_{2}+3 q q_{1}+q^{3}\right)+2 x\left(q_{1}+q^{2}\right)+q .
$$

The situation with Eq. (1.18) is similar to the Burgers equation.

\section{Acknowledgements}

F.F. would like to express his gratitude to the Department of Mathematics of Imperial College for its hospitality. A.S.F. is grateful to J. Sanders for useful suggestions. This work was partially supported by EPSRC grant No. GR/M61450 and by DGES grant PB98-0821. 


\section{Appendix}

For the convenience of the reader we summarize some known results of the theory of master symmetries. We first review two additional methods for constructing master symmetries which also rely on the existence of a scaling. We then recall the relation between master symmetries and time-dependent symmetries.

Construction 1 [5] Let $\Phi$ be a hereditary (Nijenhuis) operator [19]. Assume that $\Phi$ is invariant under the scaling $\tau_{0}$ defined by Eq. (1.9). Then $\tau=\Phi \tau_{0}$ is a master symmetry associated with

$$
\left\{q_{x}, K_{1}=\Phi q_{x}, K_{2}=\Phi^{2} q_{x}\right\}
$$

For example, the hereditary operator associated with the KdV equation (1.20) is

$$
\Phi=D_{x}^{2}+4 q+2 q_{1} \partial_{x}^{-1}
$$

This operator is invariant under the scaling $q \mapsto \alpha^{-2} q, x \mapsto \alpha x$, which gives rise to $\tau_{0}=2 q+x q_{1}$. Thus

$$
\tau=\Phi \tau_{0}=x\left(q_{3}+6 q q_{1}\right)+4 q_{2}+8 q^{2}+2 q_{1} \partial_{x}^{-1} q
$$

is a master symmetry of the $\mathrm{KdV}$ equation.

Construction 2 [4] Suppose that $K_{1}(q), K_{2}(q) \in \mathcal{R}\left\{q, \partial^{-1} q\right\}$ commute, and that $K_{2}$ admits a scaling $\tau_{0}$. Assume that there exists a function $F \in \mathcal{R}\left\{q, \partial^{-1} q, \boldsymbol{x}\right\}$ such that

$$
\tau_{0}=\left[K_{1}, F\right]_{L}
$$

Define $\tau(q) \in \mathcal{R}\left\{q, \partial^{-1} q, \boldsymbol{x}\right\}$ by

$$
\tau=\left[K_{2}, F\right]_{L}
$$

Then $\tau$ satisfies $\left[K_{1}, \tau\right]_{L}=c_{2} K_{2}$.

Indeed,

$\left[K_{1}, \tau\right]_{L}=\left[K_{1},\left[K_{2}, F\right]_{L}\right]_{L}=\left[K_{2},\left[K_{1}, F\right]_{L}\right]_{L}-\left[F,\left[K_{1}, K_{2}\right]_{L}\right]_{L}=\left[K_{2}, \tau_{0}\right]_{L}=c_{2} K_{2}$,

where the fact that $\tau_{0}$ is a scaling of $K_{2}$ has been used in the last equality.

Time-dependent symmetries. Let $\tau \in \mathcal{R}\left\{q, \partial^{-1} q, \boldsymbol{x}\right\}$ be a master symmetry associated with $\left\{q_{x}, K_{1}, K_{2}\right\}, K_{1}, K_{2} \in \mathcal{R}\left\{q, \partial^{-1} q\right\}$, normalized so that $\left[K_{1}, \tau\right]_{L}=K_{2}$. Then

$$
\sigma=t K_{2}+\tau
$$

is a time-dependent generalized symmetry of the evolution equation $q_{t}=K_{1}$. 


\section{References}

[1] A. S. Fokas and B. Fuchssteiner, The hierarchy of the Benjamin-Ono equation, Phys. Lett. 86A (1981) 341-5.

[2] H. H. Chen, Y. C. Lee and J.-E. Lin, On a new hierarchy of symmetries for the Benjamin-Ono equation, Phys. Lett. 91A (1982) 381-3.

[3] H. H. Chen, Y. C. Lee and J.-E. Lin, On a new hierarchy of symmetries for the Kadomtsev-Petviashvili equation, Physica 9D (1983) 439-45.

[4] B. Fuchssteiner, Mastersymmetries, higher order time-dependent symmetries and conserved densities of nonlinear evolution equations, Prog. Theoret. Phys. 70 (1983) 1508-22.

[5] A. S. Fokas, Symmetries and integrability, Stud. Appl. Math. 77 (1987) 253-99.

[6] I. Y. Dorfman, Dirac structures and integrability of nonlinear evolution equations, Nonlinear Science: Theory and Applications, John Wiley \& Sons, Ltd., Chichester, 1993.

[7] J. P. Zubelli and F. Magri, Differential equations in the spectral parameter, Darboux transformations and a hierarchy of master symmetries for $\mathrm{KdV}$, Commun. Math. Phys. 141 (1991) 329-51.

[8] F. A. Grünbaum and L. Haine, A theorem of Bochner, revisited, in Algebraic aspects of integrable systems, A. S. Fokas and I. M. Gel'fand (Eds.), Birkhäuser, Boston, 1997.

[9] A. V. Mikhailov and V. V. Sokolov, Integrable ODEs on associative algebras, Commun. Math. Phys. 211 (2000) 231-51.

[10] A. S. Fokas, A symmetry approach to exactly-solvable evolution equations, J. Math. Phys. 21 (1980) 1318-25.

[11] J. A. Sanders and J. P. Wang, On the integrability of homogeneous scalar evolution equations, J. Differential Equations 147 (1998) 410-34.

[12] N. K. Ibragimov and A. B. Shabat, Infinite Lie-Bäcklund algebras, Funct. Anal. Appl. 14 (1981) 313-5.

[13] F. Calogero, The evolution PDE $u_{t}=u_{x x x}+3\left(u_{x x} u^{2}+3 u_{x}^{2} u\right)+3 u_{x} u^{4}$, J. Math. Phys. 28 (1987) 538-55.

[14] P. J. Olver, Applications of Lie Groups to Differential Equations, Springer-Verlag, Berlin, 1993. 
[15] A. V. Mikhailov, A. B. Shabat and V. V. Sokolov, The symmetry approach to classification of integrable equations, in What is Integrability, V.E. Zakharov (Ed.), Springer-Verlag, Berlin, 1991.

[16] W. Oevel and B. Fuchssteiner, Explicit formulas for symmetries and conservation laws for the Kadomtsev-Petviashvili equation, Phys. Lett. 88A (1982) 323-7.

[17] K. Sawada and T. Kotera, A method for finding $N$-soliton solutions of the K.d.V. equation and K.d.V-like equation, Prog. Theoret. Phys. 51 (1974) 1355-67.

[18] B. Fuchssteiner and W. Oevel, The bi-Hamiltonian structure of some nonlinear fifth- and seventh-order differential equations and recursion formulas for their symmetries and conserved covariants, J. Math. Phys. 23 (1982) 358-63.

[19] B. Fuchssteiner, Application of hereditary symmetries to nonlinear evolution equations, Nonlinear Anal. Theory Meth. Appl. 3 (1979) 849-62. 